\title{
Perfil epidemiológico das fissuras labiopalatais de crianças atendidas em um centro de referência paranaense
}

\author{
Epidemiological profile of labiopalatine clefts in children cared for at a reference center in \\ Paraná \\ Perfil epidemiológico de las hendiduras labiopalatinas en niños tratados en un centro de \\ referencia en Paraná
}

\section{Fabiana Gonçalves de Oliveira Azevedo Matos ${ }^{\mathrm{I}}$, Kelly Jackelini Jorge dos Santos ${ }^{\mathrm{II}}$ Mariângela Monteiro Melo Baltazar ${ }^{I I I}$, Carlos Alexandre Molena Fernandes ${ }^{\text {IV }}$ Aline Fernanda Junges Marques ${ }^{\mathrm{v}}$, Mateus Souza da Luz ${ }^{\mathrm{VI}}$}

\begin{abstract}
Resumo: Objetivo: analisar o perfil epidemiológico das fissuras labiopalatais de crianças atendidas em um centro de atenção e pesquisa em anomalias craniofaciais. Método: estudo epidemiológico, documental, transversal, que avaliou 116 crianças, com idade de zero a até 12 anos incompletos, com fissura labial e/ou palatina atendidas em um centro de referência do oeste do Paraná de fevereiro de 2013 a fevereiro de 2017 Resultados: houve predominância de pacientes do sexo masculino ( $\mathrm{n}=67$; $57,8 \%$ ), residentes em Cascavel $(\mathrm{n}=58 ; 50 \%)$, com mais que 5 anos no momento da primeira consulta $(n=41 ; 35,3 \%)$. As fissuras mais frequentes eram de extensão completa $(n=56 ; 50,9 \%)$, do tipo labiopalatina $(n=50 ; 43,1 \%)$ e unilaterais à esquerda $(n=35 ; 44,3 \%)$. Conclusões: os dados obtidos estão de acordo com os achados da literatura e evidenciam a necessidade de medidas que viabilizem o acesso precoce das crianças fissuradas ao referido serviço especializado.
\end{abstract}

Descritores: Saúde da criança; Fenda labial; Fissura palatina; Epidemiologia; Perfil de saúde

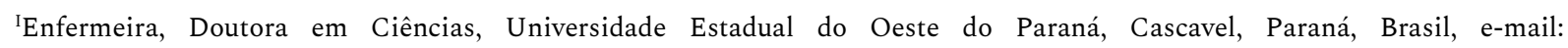
fabianamatos@hotmail.com, ORCID: https://orcid.org/0000-0002-5283-5363

IIEnfermeira, Mestre em Enfermagem, Hospital Universitário do Oeste do Paraná, Cascavel, Paraná, Brasil, e-mail: kellyjack@hotmail.com, ORCID: https://orcid.org/0000-0003-1452-3606

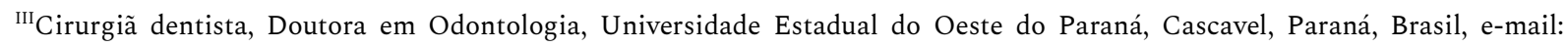
mmmwgb@uol.com.br, ORCID: https://orcid.org/0000-0001-6395-9516

${ }^{\mathrm{IV}}$ Enfermeiro, Doutor em Ciências Farmacêuticas, Universidade Estadual de Maringá, Maringá, Paraná, Brasil, e-mail: carlosmolena126@gmail.com, ORCID: https://orcid.org/0000-0002-4019-8379

${ }^{\vee}$ Assistente social, Hospital Universitário do Oeste do Paraná, Cascavel, Paraná, Brasil, e-mail: alinejunges@yahoo.com.br, ORCID: https://orcid.org/0000-0002-0684-8403

${ }^{\mathrm{VI}}$ Enfermeiro, Especialista em Gerenciamento de Enfermagem em Clínica Médica e Cirúrgica, Universidade Estadual do Oeste do Paraná, Cascavel, Paraná, Brasil, e-mail: matthheus72@gmail.com, ORCID: https://orcid.org/0000-0003-2405-0152
} 


\begin{abstract}
Objective: to analyze the epidemiological profile of labiopalatine clefts in children cared for at a care and research center on craniofacial anomalies. Method: this is an epidemiological, documentary and cross-sectional study that assessed 116 children, aged from 0 to 12 incomplete years, with labial and/or palatine cleft cared for at a reference center in western Paraná from February 2013 to February 2017 Results: there was a predominance of male patients ( $\mathrm{n}=67 ; 57.8 \%$ ), living in Cascavel $(n=58 ; 50 \%)$ and over five years old at the time of the first consultation $(n=41 ; 35.3 \%)$. The most frequent clefts were typified as: complete extension ( $n=56 ; 50.9 \%)$, labiopalatine $(n=50 ; 43.1 \%)$ and left-sided unilateral $(\mathrm{n}=35 ; 44.3 \%)$. Conclusions: the acquired data are in accordance with the findings of the pertinent literature and show the need for measures that enable the early access of cleft children to this specialized service.
\end{abstract}

Descriptors: Child health; Cleft lip; Cleft palate; Epidemiology; Health profile

Resumen: Objetivo: analizar el perfil epidemiológico de las hendiduras labiopalatinas en niños tratados en un centro de atención e investigación sobre anomalías craneofaciales. Método: estudio epidemiológico, documental, transversal que evaluó a 116 niños, de 0 a 12 años de edad, con labio leporino y/o palatino, tratados en un centro de referencia en el oeste de Paraná desde febrero de 2013 hasta febrero de 2017 Resultados: hubo un predominio de pacientes masculinos ( $\mathrm{n}=67 ; 57.8 \%$ ), residentes en Cascavel $(n=58 ; 50 \%)$ y mayores de 5 años en el momento de la primera consulta $(n=41$; 35.3\%). Las fisuras más frecuentes fueron de extensión completa $(\mathrm{n}=56 ; 50,9 \%)$, del tipo labiopalatino ( $n=50 ; 43,1 \%)$ y unilaterales a la izquierda $(n=35 ; 44,3 \%)$. Conclusiones: los datos obtenidos están en línea con los hallazgos de la literatura y muestran la necesidad de medidas que permitan el acceso temprano de los niños con hendiduras a ese servicio especializado.

Descriptores: Salud del niño; Labio leporino; Fisura del paladar; Epidemiología; Perfil de salud

\title{
Introdução
}

As fissuras de lábio e/ou palato são alterações que resultam de falhas na fusão dos processos de formação nasal e da maxila, sendo consideradas as anomalias congênitas faciais mais frequentes. ${ }^{1}$ Tais alterações possuem ampla variação sendo as fissuras de lábio, de palato, de lábio e palato, uni ou bilaterais as mais comuns, podendo ser encontradas de forma isolada ou associadas com outras alterações, como parte de alguma síndrome. ${ }^{2} \mathrm{~A}$ nível mundial a incidência de fissura labiopalatal é de 0,5 a 2 casos a cada mil nascidos vivos, e no Brasil, a incidência é de um caso a cada 650 nascidos vivos. ${ }^{3}$

Os pacientes com fissuras labiopalatais, assim como seus familiares enfrentam diversos obstáculos funcionais, psicológicos e sociais frente ao 
3 | Matos FGOA, Santos KJJ, Baltazar MMM, Fernandes CAM, Marques AFJ, Luz MS

diagnóstico de má formação. O tratamento dos mesmos deve ser realizado em um âmbito interdisciplinar especializado, visando reabilitação estética, funcional (sucção, deglutição, mastigação, respiração, fonação, audição) e psicossocial do indivíduo. ${ }^{3}$ Nesse contexto, a interação da equipe multiprofissional de saúde com a família e com o paciente constitui ação indis pensável para o sucesso da reabilitação. ${ }^{4}$

Conhecer o perfil epidemiológico dos pacientes atendidos otimiza as ações das equipes de saúde que os assistem, pois permite reavaliar e direcionar as condutas a serem realizadas, possibilitando melhor estruturação do serviço. A questão de pesquisa foi: qual o perfil epidemiológico das fissuras labiopalatais de crianças atendidas em um centro de referência do oeste do Paraná? Dessa forma, o presente estudo objetivou analisar o perfil epidemiológico das fissuras labiopalatais de crianças atendidas em um centro de atenção e pesquisa em anomalias craniofaciais.

\section{Método}

Trata-se de um estudo epidemiológico, documental e retrospectivo. A pesquisa foi desenvolvida no Centro de Atenção e Pesquisa em Anomalias Craniofaciais (CEAPAC), localizado anexo ao Hospital Universitário do Oeste do Paraná (HUOP). O referido serviço, inaugurado no início de 2013, oferece tratamento especializado para os pacientes com fissuras labiopalatais, provenientes principalmente da Região Oeste, Sudoeste e Noroeste do Paraná (PR), assim como, aqueles provenientes do Estado de Santa Catarina (SC), Mato Grosso do Sul (MS) e do Paraguai (região fronteiriça).

O Estado do Paraná conta, até o momento, com três centros de referência para o tratamento de pacientes fissurados sendo eles: o Centro de Atendimento Integral ao Fissurado Labiopalatal (CAIF), localizado na cidade de Curitiba, o Centro 
Perfil epidemiológico das fissuras labiopalatais de crianças atendidas em um... | 4 de Apoio e Reabilitação dos Portadores de Fissura Labiopalatal (CEFIL), localizado na cidade de Londrina e o CEAPAC, localizado em Cascavel. De fevereiro de 2013 a fevereiro de 2017 foram cadastrados no referido serviço um total de 819 pacientes, sendo realizados 11.132 atendimentos ambulatoriais e 31 cirurgias. Desse total, 4.778 atendimentos e 19 procedimentos cirúrgicos foram realizados em crianças de até 12 anos de idade. Fizeram parte do estudo 116 crianças que atendiam os seguintes critérios de seleção: ter fissura labial e/ou palatal, ter de zero a até 12 anos incompletos (11 anos, 11 meses e 29 dias) no momento da primeira consulta no referido serviço e ter sido atendida de fevereiro de 2013 a fevereiro de 2017.

As variáveis do estudo foram: sexo; idade na primeira consulta; procedência; particularidades da fissura [tipo de fissura: labial (pré-forame), labiopalatina (transforame ou pré/pós-forame) e palatina (submucosa ou pós-forame); extensão da fissura: completa, incompleta e mistas; e lateralidade da fissura: direita, esquerda e bilateral]. As particularidades da fissura foram classificadas segundo as autoras com base na classificação de Spina. ${ }^{2}$

Os dados foram coletados dos prontuários físico e eletrônico (Tasy $\left.{ }^{\circledR}\right)$ do referido serviço, digitados em planilha do programa Microsoft Office Excel 2013 versão 20 e exportados para o software $\mathrm{R}$, versão 3.6.0, para realização das análises estatísticas. Para verificar a associação estatística entre as variáveis de estudo foram realizados o teste de Qui-quadrado e o teste exato de Fisher, considerando nível de significância de $5 \%(\alpha<0,05)$.

A pesquisa foi aprovada pelo Comitê Permanente de Ética em Pesquisa com Seres Humanos da Universidade Estadual de Maringá, parecer n: 2.794.738/2018, CAAE: 85143717.0.0000.0104, aprovado em 01/08/2018.

\section{Resultados}


Dos 116 pacientes avaliados (100\%), 57,8\% ( $\mathrm{n}=67)$ eram do sexo masculino; $50 \%$ $(n=58)$ residiam em Cascavel e $35,3 \%(n=41 ;)$ tinham mais de cinco anos no momento da primeira consulta no CEAPAC. As fissuras mais frequentemente identificadas no estudo eram de extensão completa ( $n=56 ; 50,9 \%)$, unilaterais à esquerda $(n=35 ; 44,3 \%)$ e do tipo labiopalatina $(\mathrm{n}=50 ; 43,1)$ (Tabela 1$)$.

Tabela 1 - Variáveis de estudo das crianças com fissuras labiopalatais atendidas no CEAPAC/HUOP no período de fevereiro de 2013 a fevereiro de 2017. Cascavel, 2018.

\begin{tabular}{|c|c|c|}
\hline Variáveis & $\mathbf{N}$ & $\%$ \\
\hline \multicolumn{3}{|l|}{ Sexo* } \\
\hline Masculino & 67 & 57,8 \\
\hline Feminino & 49 & 42,2 \\
\hline \multicolumn{3}{|l|}{ Procedência* } \\
\hline Cascavel & 58 & 50,0 \\
\hline Outros municípios & 58 & 50,0 \\
\hline \multicolumn{3}{|c|}{ Idade $1^{\circ}$ atendimento (meses) ${ }^{*}$} \\
\hline $0-11$ & 39 & 33,6 \\
\hline $12-23$ & 07 & 6,1 \\
\hline $24-59$ & 29 & 25,0 \\
\hline $60-144$ & 41 & 35,3 \\
\hline \multicolumn{3}{|l|}{ Tipo de Fissura* } \\
\hline Labiopalatina & 50 & 43,1 \\
\hline Labial & 30 & 25,9 \\
\hline Palatina & 36 & 31,0 \\
\hline \multicolumn{3}{|l|}{ Extensão da Fissura ${ }^{* *}$} \\
\hline Completa & 56 & 50,9 \\
\hline Incompleta & 50 & 45,5 \\
\hline Mista & 04 & 3,6 \\
\hline \multicolumn{3}{|l|}{ Lateralidade da Fissura $^{* \star *}$} \\
\hline Esquerda & 35 & 44,3 \\
\hline Direita & 25 & 31,6 \\
\hline Bilateral & 19 & 24,1 \\
\hline
\end{tabular}

*Tamanho da amostra: $\mathrm{n}=116$

${ }^{* *}$ Tamanho da amostra: $\mathrm{n}=110$

*** Tamanho da amostra: $\mathrm{n}=79$ 
Perfil epidemiológico das fissuras labiopalatais de crianças atendidas em um... 6

As análises estatísticas mostraram que não houve associação entre o sexo e a procedência das crianças com as particularidades das fissuras

(Tabela 2).

Tabela 2 - Variáveis demográficas e particularidades das fissuras das crianças com fissuras labiopalatais atendidas no CEAPAC/HUOP no CEAPAC/HUOP no período de fevereiro de 2013 a fevereiro de 2017. Cascavel, 2018.

\begin{tabular}{|c|c|c|c|c|c|c|c|c|c|c|c|c|c|c|c|c|c|c|c|c|c|c|c|c|}
\hline \multirow{4}{*}{ Variáveis } & \multicolumn{24}{|c|}{ Diagnóstico da Fissura } \\
\hline & \multicolumn{8}{|c|}{ Tipo* $^{*}$} & \multicolumn{8}{|c|}{ Extensão ${ }^{* *}$} & \multicolumn{8}{|c|}{ Lateralidade $^{* * *}$} \\
\hline & \multicolumn{2}{|c|}{ Labiopalatal } & \multicolumn{2}{|c|}{ Palatina } & \multicolumn{2}{|c|}{ Labial } & \multirow[t]{2}{*}{ Total } & \multirow[t]{2}{*}{$\mathbf{p} \S$} & \multicolumn{2}{|c|}{ Completa } & \multicolumn{2}{|c|}{ Incompleta } & \multicolumn{2}{|c|}{ Mista } & \multirow[t]{2}{*}{ Total } & \multirow[t]{2}{*}{$\mathbf{p} \S$} & \multicolumn{2}{|c|}{ Esquerda } & \multicolumn{2}{|c|}{ Direita } & \multicolumn{2}{|c|}{ Bilateral } & \multirow[t]{2}{*}{ Total } & \multirow[t]{2}{*}{$\mathbf{p} \S$} \\
\hline & $\mathrm{n}$ & $\%$ & $\mathrm{n}$ & $\%$ & $\mathrm{n}$ & $\%$ & & & $\mathrm{n}$ & $\%$ & $\mathrm{n}$ & $\%$ & $\mathrm{n}$ & $\%$ & & & $\mathrm{n}$ & $\%$ & $\mathrm{n}$ & $\%$ & $\mathrm{n}$ & $\%$ & & \\
\hline \multicolumn{25}{|l|}{ Sexo } \\
\hline Masculino & 31 & 46,3 & 17 & 25,4 & 19 & 28,4 & 67 & \multirow{2}{*}{0,3029} & 33 & 49,3 & 31 & 46,3 & 3 & 4,5 & 67 & \multirow{2}{*}{0,7982} & 22 & 44,9 & 13 & 26,5 & 14 & 28,6 & 49 & \multirow[b]{2}{*}{0,3373} \\
\hline Feminino & 19 & 38,8 & 19 & 38,8 & 11 & 22,4 & 49 & & 23 & 53,5 & 19 & 44,2 & 1 & 2,3 & 43 & & 13 & 43,3 & 12 & 40,0 & 5 & 16,7 & 30 & \\
\hline \multicolumn{25}{|l|}{ Procedência } \\
\hline Cascavel & 20 & 34,4 & 19 & 32,8 & 19 & 32,8 & 58 & & 23 & 42,6 & 29 & 53,7 & 2 & 3,7 & 54 & \multirow{3}{*}{0,2198} & 18 & 46,2 & 15 & 38,5 & 6 & 15,4 & 39 & \multirow{3}{*}{0,1580} \\
\hline Demais & & & & & & & & 0,1198 & & & & & & & & & & & & & & & & \\
\hline municípios & 30 & 51,7 & 17 & 29,3 & 11 & 19,0 & 58 & & 33 & 58,9 & 21 & 37,5 & 2 & 3,6 & 56 & & 17 & 42,5 & 10 & 25,0 & 13 & 32,5 & 40 & \\
\hline
\end{tabular}

$\S$ Teste do qui-quadrado

*Tamanho da amostra: $\mathrm{n}=116$

** Tamanho da amostra: $\mathrm{n}=110$

${ }^{* * *}$ Tamanho da amostra: $\mathrm{n}=79$ 
O estudo também não identificou associação entre as variáveis demográficas (sexo e procedência) e as particularidades das fissuras (tipo, extensão e lateralidade) com a idade da criança no primeiro atendimento realizado no CEAPAC (Tabela 3).

Tabela 3 - Variáveis demográficas e particularidades das fissuras das crianças com fissuras labiopalatais 0de acordo com a idade no primeiro atendimento realizado no CEAPAC/HUOP, no período de fevereiro de 2013 a fevereiro de 2017. Cascavel, 2018.

\begin{tabular}{|c|c|c|c|c|c|c|c|c|c|c|}
\hline \multirow{3}{*}{ Variáveis } & \multicolumn{8}{|c|}{ Idade na Primeira Consulta (meses) } & \multirow{3}{*}{ Total } & \multirow{3}{*}{ p§ } \\
\hline & \multicolumn{2}{|c|}{$0-11$} & \multicolumn{2}{|c|}{$12-23$} & \multicolumn{2}{|c|}{$24-59$} & \multicolumn{2}{|c|}{$60-144$} & & \\
\hline & $\mathrm{n}$ & $\%$ & $\mathrm{n}$ & $\%$ & $\mathrm{n}$ & $\%$ & $\mathrm{n}$ & $\%$ & & \\
\hline \multicolumn{11}{|l|}{ Sexo* } \\
\hline Masculino & 25 & 37,3 & 3 & 4,5 & 15 & 22,4 & 24 & 35,8 & 67 & 0,6850 \\
\hline Feminino & 14 & 28,6 & 4 & 8,2 & 13 & 26,5 & 18 & 36,7 & 49 & \\
\hline \multicolumn{11}{|l|}{ Procedência* } \\
\hline Cascavel & 13 & 22,4 & 4 & 6,9 & 18 & 31,0 & 23 & 39,7 & 58 & 0,0794 \\
\hline Demais municípios & 26 & 44,8 & 3 & 5,2 & 11 & 19,0 & 18 & 31,0 & 58 & \\
\hline \multicolumn{11}{|l|}{ Tipo de Fissura* } \\
\hline Labiopalatina & 18 & 36,0 & 3 & 6,0 & 12 & 24,0 & 17 & 34,0 & 50 & \\
\hline Labial & 7 & 23,3 & 2 & 6,7 & 7 & 23,3 & 14 & 46,7 & 30 & 0,8490 \\
\hline Palatina & 14 & 38,9 & 2 & 5,6 & 9 & 25,0 & 11 & 30,5 & 36 & \\
\hline \multicolumn{11}{|l|}{ Extensão da Fissura** } \\
\hline Completa & 20 & 35,7 & 3 & 5,4 & 14 & 25,0 & 19 & 33,9 & 56 & \\
\hline Incompleta & 16 & 32,0 & 3 & 6,0 & 12 & 24,0 & 19 & 38,0 & 50 & 0,6433 \\
\hline Mista & 2 & 50,0 & 1 & 25,0 & 1 & 25,0 & 0 & 0,0 & 4 & \\
\hline \multicolumn{11}{|c|}{ Lateralidade da Fissura $^{* * *}$} \\
\hline Esquerda & 8 & 22,9 & 4 & 11,4 & 8 & 22,9 & 15 & 42,9 & 35 & \\
\hline Direita & 8 & 32,0 & 1 & 4,0 & 7 & 28,0 & 9 & 36,0 & 25 & 0,4347 \\
\hline Bilateral & 9 & 47,4 & 0 & 0,0 & 4 & 21,1 & 6 & 31,6 & 19 & \\
\hline
\end{tabular}

§Teste do qui-quadrado

*Tamanho da amostra: $\mathrm{n}=116$

**Tamanho da amostra: $\mathrm{n}=110$

*** Tamanho da amostra: $\mathrm{n}=79$

\section{Discussão}

$\mathrm{Na}$ amostra de estudo avaliada $(\mathrm{n}=116 ; 100 \%)$ houve predominância de pacientes do sexo masculino $(n=67 ; 57,8 \%)$, residentes em Cascavel $(n=58 ; 50 \%)$ e com mais de cinco anos no momento da primeira consulta no CEAPAC ( $\mathrm{n}=41 ; 35,3 \%)$ (Tabela 1$)$. O maior número de pacientes fissurados do sexo masculino condiz com os achados da literatura. ${ }^{5-13} \mathrm{O}$ fato de a 
metade dos pacientes avaliados $(n=58 ; 50 \%)$ residirem em Cascavel não quer dizer que o número de pacientes com fissuras labiopalatais seja maior nessa região. Esse achado pode ser explicado pela facilidade de acesso dos mesmos ao CEAPAC e pela possibilidade dos demais pacientes com fissuras labiopalatais nascidos no interior do Paraná serem encaminhados para os outros serviços de referência localizados no Estado (CAIF e CEFIL).

O cumprimento das atuais políticas públicas de atenção à saúde ${ }^{14-15}$ que determinam a criação e o credenciamento de novos centros de atendimento especializado aos pacientes com anomalias craniofaciais deve favorecer o amplo acesso dessa população aos serviços de prevenção, tratamento e reabilitação. A chegada tardia das crianças para tratamento no CEAPAC (com idade superior a cinco anos) pode estar relacionada ao pouco tempo de funcionamento do mesmo. Como mencionado anteriormente, o CEAPAC foi inaugurado em fevereiro de 2013 e a grande maioria dos pacientes atendidos já havia iniciado o seu tratamento em outros centros de saúde especializados.

Assim como evidenciado pela literatura, o presente estudo identificou predominância de fissuras do tipo labiopalatina $(n=50 ; 43,1 \%)^{6,8-12,16-20}$ de extensão completa $(n=56 ; 50,9 \%)^{6,10,19}$ e unilaterais à esquerda $(n=35 ; 44,3 \%)$. $6,8,10,12,17,21$ Conhecer o perfil das fissuras predominantes no serviço contribui para a otimização do plano terapêutico multiprofissional, visto que, quanto mais complexa é a lesão, maior é o comprometimento funcional do paciente assistido. ${ }^{10}$

Ainda não há consenso na literatura sobre a causa da prevalência da fissura no lado esquerdo. Uma tentativa de explicação seria o maior aporte sanguíneo na hemiface direita durante o período embrionário devido a pressão sanguínea exercida pela carótida interna direita. ${ }^{18}$ Outra hipótese seria a proximidade da região afetada com o coração durante o período embrionário, que poderia aumentar o risco da formação de fissura devido o batimento do referido órgão. ${ }^{12}$ 
O estudo não identificou associação entre o sexo e a procedência das crianças com as particularidades das fissuras. Embora sem significância estatística, os indivíduos do sexo masculino apresentaram as maiores proporções de fissura labiopalatina $(n=31 ; 46,3 \%),{ }^{6,8-10,20}$ de extensão completa $(n=33 ; 49,3 \%),{ }^{6,18}$ e com lateralidade a esquerda ( $\left.n=22 ; 44,9 \%\right) .6,20$ Também sem significância estatística, os pacientes nascidos em Cascavel-PR apresentaram maiores proporções de fissura de extensão incompleta $(n=29 ; 53,7 \%)$, de lateralidade a esquerda $(n=18$; $46,2 \%)$ e do tipo labiopalatina $(n=20 ; 34,4 \%)$ (Tabela 2$)$.

Embora não se tenha observado associação estatística entre as variáveis estudadas (Tabela 3), é possível fazer algumas considerações a esse respeito. Com relação à procedência, observa-se que a metade $(n=58 ; 50 \%)$ das crianças com fissuras labiopalatais atendidas no CEAPAC era proveniente de Cascavel-PR. Tal fato pode ser explicado devido a exposição das gestantes à agentes poluentes, comuns nos grandes centros urbanos, podendo contribuir para o nascimento de crianças com fissuras. ${ }^{21}$ A outra metade da amostra de estudo ( $\left.\mathrm{n}=58 ; 50 \%\right)$ era proveniente de outros municípios de menor porte, com economia essencialmente agrícola. Da mesma forma que a poluição, os agrotóxicos podem favorecer a ocorrência de malformações congênitas nas áreas rurais. ${ }^{22}$

Os dados mostram que os municípios vizinhos a Cascavel ( $\mathrm{n}=26 ; 44,8 \%)$ tendem a encaminhar as crianças ao CEAPAC mais precocemente (no primeiro ano de vida) quando comparados com as crianças procedentes de Cascavel $(n=13 ; 22,4 \%)$ (Tabela 3$)$. Tal achado pode ser explicado pelo fato de as pequenas cidades não disporem de serviços assistenciais que dão suporte às crianças com necessidades especiais logo nos primeiros meses de vida, fazendo com que as mesmas sejam encaminhadas para os grandes centros mais precocemente.

Apesar de contraditório, o fato de a maioria das crianças nascidas em Cascavel ( $\mathrm{n}=23$; $39,7 \%$ ) demorarem mais para iniciar o tratamento no CEAPAC (após cinco anos de vida), pode ser explicado pela variedade de opções assistenciais de saúde disponíveis na cidade, mesmo que 
não especializados. Outra hipótese seria que, por ser um serviço de saúde novo (com menos de quatro anos de funcionamento no momento da coleta dos dados de pesquisa), grande parte dos pacientes atendidos no CEAPAC já haviam iniciado o tratamento em outros centros de referência.

Embora sem associação estatística, os pacientes com fissuras do tipo labiopalatina ( $\mathrm{n}=18 ; 36,0 \%)$ e palatina $(14 ; 38,9 \%)$, classificadas como mista (pré e pós-forame) ( $\mathrm{n}=2 ; 50,0 \%)$ e bilateral $(\mathrm{n}=9 ; 47,4 \%)$ tendem a chegar ao serviço especializado ainda no primeiro ano de vida. Uma justificativa para isso seria a complexidade das referidas alterações que podem oferecer risco à manutenção da vida da criança devido a dificuldade no processo de alimentação. ${ }^{23}$ Seguindo esse mesmo raciocínio, é coerente considerar que devido a menor gravidade da alteração craniofacial, as crianças com fissura do tipo labial $(\mathrm{n}=14 ; 46,7 \%)$ tendem a chegar mais tardiamente aos centros de referência (após cinco anos de vida).

A idade preconizada para o início do tratamento do paciente fissurado é entre um mês e dois anos de idade devido o tempo cirúrgico ideal para a realização das cirurgias primárias de lábio e de palato. ${ }^{10,24} \mathrm{~A}$ busca precoce pelo tratamento favorece a obtenção de resultados estéticos e funcionais mais satisfatórios devido a condução adequada do plano terapêutico. ${ }^{25}$ É recomendado a ampla divulgação dos serviços especializados com a finalidade de dar suporte físico, emocional e social tanto ao indivíduo com anomalias crânio faciais quanto à sua família. ${ }^{13}$

\section{Conclusões}

O estudo identificou predominância de fissuras labiopalatais em pacientes do sexo masculino ( $\mathrm{n}=67 ; 57,8 \%)$, residentes em Cascavel $(\mathrm{n}=58 ; 50 \%)$, com mais de cinco anos no momento da primeira consulta $(\mathrm{n}=41 ; 35,3 \%)$. As fissuras mais frequentes eram de extensão completa ( $n=56 ; 50,9 \%)$, do tipo labiopalatina $(n=50 ; 43,1 \%)$ e unilaterais à esquerda $(n=35 ; 44,3 \%)$. 
Ter conhecimento sobre o perfil epidemiológico das fissuras labiopalatais dos pacientes assistidos permite o planejamento de ações pontuais que buscam atender as particularidades dessa população. O acesso tardio das crianças com fissura labiopalatal no serviço pesquisado reforça a necessidade de medidas que viabilizem a admissão precoce das mesmas nos centros especializados uma vez que a adequada condução inicial do tratamento propicia resultados estéticos, funcionais e psicossociais mais satisfatórios.

Destaca-se que o pequeno número de pacientes que compôs a amostra de estudo poderia ter sido considerada um fator de limitação da pesquisa, no entanto, o tamanho da amostra correspondeu à totalidade das crianças atendidas no período de coleta de dados. Novas pesquisas envolvendo pacientes com outras faixas etárias poderão auxiliar na condução do tratamento dos indivíduos com tais alterações craniofaciais.

\section{Referências}

1. Borges-Osório MR, Robinson WM. Genética humana. 3르 ed. Porto Alegre (RS): Artmed; 2013.

2. Spina V, Psillakis JM, Lapa FS, Ferreira MC. Classificação das fissuras lábio-palatais: sugestão de modificação. Rev Hosp Clin Fac Med Univ São Paulo. 1972;27(1):5-6.

3. Tanikawa D, Alonso N. Fissuras Labio Palatais. In: Gemperli R, Munhoz AM, Marques Neto AZ. Fundamentos da cirurgia plástica. Rio de Janeiro (RJ): Thieme; 2015. Cap. 12.

4. Silva DP, Dornelles S, Paniagua LM, Costa SS, Collares MVM. Aspectos patofisiológicos do esfíncter velofaríngeo nas fissuras palatinas. Arq Int Otorrinolaringol [Internet]. 2008 [acesso em 2018 set 10];12(3):426-35. Disponível em: http://www.arquivosdeorl.org.br/conteudo/pdfForl/551.pdf

5. Cunha ECM, Fontana R, Fontana T, Silva WR, Moreira QVP, Garcias GL, et al. Antropometria e fatores de risco em recém-nascidos com fendas faciais. Rev Bras Epidemiol [Internet]. 2004 [acesso em 2004 set 20];7:417-22. Disponível em: http://www.scielo.br/pdf/rbepid/v7n4/05.pdf

6. Coutinho AL, Lima MC, Kitamura MAP, Neto JF, Pereira RM. Perfil epidemiológico dos portadores de fissuras orofaciais atendidos em um Centro de Referência do Nordeste do Brasil. Rev Bras Saúde Mater Infant [Internet]. 2009 [acesso em 2018 out 10];9(2):149-56. Disponível em: http://www.scielo.br/pdf/rbsmi/v9n2/a04v9n2.pdf 
7. Menezes LM, Rizzatto SMD, Azeredo FVDA. Characteristics and distribution of dental anomalies in a Brazilian cleft population. Rev Odonto Ciênc [Internet]. 2010 [acesso em 2018 set 15];25(2):137-41. Disponível em: http://www.scielo.br/pdf/roc/v25n2/06.pdf

8. Cymrot M, Sales FCD, Teixeira FAA, Teixeira Junior FAA, Teixeira GSB, Cunha Filho JF, et al. Prevalência dos tipos de fissura em pacientes com fissuras labiopalatinas atendidos em um Hospital Pediátrico do Nordeste brasileiro. Rev Bras Cir Plást [Internet]. 2010 [acesso em 2018 set 23];25(4):648-51. Disponível em: http://dx.doi.org/10.1590/S1983-51752010000400015

9. Di Ninno CQMS, Fonseca LFN, Pimenta MVE, Vieira ZG, Fonseca JA, Miranda ICC, et al. Levantamento epidemiológico dos pacientes portadores de fissura de lábio e/ou palato de um centro especializado de Belo Horizonte. Rev CEFAC [Internet]. 2011 [acesso em 2018 out 20];13(6):1002-8. Disponível em: http://dx.doi.org/10.1590/S1516-18462011005000046

10. Gardenal M, Bastos PRHO, Pontes ERJC, Bogo D. Prevalência das fissuras orofaciais diagnosticadas em um serviço de referência em casos residentes no estado de Mato Grosso do Sul. Arquivos Int Otorrinolaringol [Internet]. 2011 [acesso em 2018 out 23];15(2):133-41. Disponível em: http://dx.doi.org/10.1590/S1809-48722011000200003

11. Figueirêdo CJR, Vasconcelos WKS, Maciel SSSV, Maciel WV, Gondim LAM, Tassitano RM. Prevalência de fissuras orais no Estado do Rio Grande do Norte, Brasil, entre 2000 e 2005. Rev Paul Pediatr [Internet]. 2011 [acesso em 2018 out 23];29(1):29-34. Disponível em: http://dx.doi.org/10.1590/S0103-05822011000100005

12. Rebouças PD, Moreira MM, Chagas, MLB, Cunha Filho JF. Prevalência de fissuras labiopalatinas em um hospital de referência do nordeste do Brasil. Rev Bras Odontol [Internet]. 2014 [acesso em 2018 out 25];71(1):39-41. Disponível em: http://revista.aborj.org.br/index.php/rbo/article/viewFile/487/401

13. Xavier KM, Britto DBO, Di Ninno CQMS. Fissura palatina: prevalência regional no estado de Minas Gerais em centro especializado referencial. Rev Méd Minas Gerais [Internet]. 2015 [acesso em 2018 out 25];25(2):157-61. Disponível em: http://rmmg.org/exportar-pdf/1768/v25n2a03.pdf

14. BRASIL. Ministério da Saúde. Portaria no 126 SAS/MS, de 17 de setembro de 1993. Cria grupos e procedimentos para tratamento de lesões labiopalatais na tabela SIH/SUS e dá outras providências. Diário Oficial da União, Brasília (DF); 21 set 1993. Seção 1, p. 12.

15. Ministério da Saúde (BR). Reduzindo as desigualdades e ampliando o acesso à assistência à saúde no Brasil 1998-2002. Brasília (DF): Ministério da Saúde; 2002.

16. Freitas MCA, Batista TS, Pereira MCG, Brandão MM, Marianetti LVS, Almeida PP. Estudo epidemiológico das fissuras labiopalatinas na Bahia. Rev Uningá [Internet]. 2018 [acesso em 2018 out 25];37(1):13-22. Disponível em: http://revista.uninga.br/index.php/uninga/article/view/1127 
17. Amstalden-Mendes LG, Xavier AC, Antunes DK, Ferreira ACRG, Tonocchi R, Fett-conte AC, et al. Estudo multicêntrico da época do diagnóstico de fendas orais. J Pediatr (Rio J) [Internet]. 2011 [acesso em 2018 out 23];87(3):225-30. Disponível em: http://www.scielo.br/scielo.php?pid=S0021$75572011000300008 \&$ script=sci_abstract $\&$ tlng=pt

18. Freitas JAS, Dalben GS, Santamaria JM, Freitas PZ. Informações atuais sobre a caracterização das fissuras orofaciais no Brasil. Braz Oral Res. 2004;18(2):128-33.

19. Loffredo LCM, Freitas JAS, Grigolli AAG. Prevalência de fissuras orais de 1975 a 1994. Rev Saúde Pública [Internet]. 2001 [acesso em 2018 out 25];35(6):571-5. Disponível em: http://www.scielo.br/scielo.php?script=sci_arttext\&pid=S0034-89102001000600011

20. Magdalenic-Mestrovic M, Bagatin M. An epidemiological study of orofacial clefts in Croatia 19881998. J Craniomaxillofac Surg [Internet]. 2005 [acesso em 2018 set 23];33(2):85-90. Disponível em: https://www.ncbi.nlm.nih.gov/pubmed/15804585

21. Loffredo LCM, Souza JMP, Yunes J, Freitas JAS, Spiri WC. Fissuras labiais: estudo caso-controle. Rev Saúde Pública [Internet]. 1994 [acesso em 2018 out 25];28(3):213-7. Disponível em: http://www.scielo.br/scielo.php?pid=S0034-89101994000300009\&script=sci_abstract\&tlng=pt

22. Dutra LS, Ferreira AP. Associação entre malformações congênitas e a utilização de agrotóxicos em monoculturas no Paraná, Brasil. Saúde Debate [Internet]. 2017 [acesso em 2018 out 08];41(N Esp 2):241-53. Disponível em: http://www.scielo.br/scielo.php?pid=S0103$11042017000600241 \&$ script=sci_abstract\&tlng=pt

23. Beluci ML, Barros SP, Fontes CMB, Trettene A, Mondini CCSD. Nursing diagnoses and interventions in postoperative alveolar bone graft patients. Rev Enferm UERJ [Internet]. 2017 [acesso em 2020 fev 20];25:e19872. Disponível em: https://www.epublicacoes.uerj.br/index.php/enfermagemuerj/article/view/19872/24272

24. Silva Filho OG, Freitas JAS, Okada T. Fissuras labiopalatais: diagnóstico e uma filosofia interdisciplinar de tratamento. In: Pinto VG. Saúde Bucal Coletiva. $4^{\mathbf{a}}$ ed. São Paulo (SP): Santos; 2000. p. 480-527.

25. Melgaço CA, Di Ninno CQMS, Penna LM, Vale MPP. Aspecto ortodôntico/ortopédicos e fonoaudiológicos relacionados a pacientes portadores de fissuras labiopalatinas. J Bras Ortodon Ortop Facial [Internet]. 2002 [acesso em 2018 out 10];7(37):23-32. Disponível em: http://pesquisa.bvs.br/brasil/resource/pt/lil-317097 


\section{Autor correspondente}

Fabiana Gonçalves de Oliveira Azevedo Matos

E-mail: fabianamatos@hotmail.com

Endereço: Rua Monjoleiro, 125, Tropical, Cascavel-PR

CEP: 85807-300.

\section{Contribuições de Autoria}

\section{1 - Fabiana Gonçalves de Oliveira Azevedo Matos}

Participação ativa na concepção e planejamento do projeto de pesquisa, obtenção, análise e interpretação dos dados, redação e revisão crítica do manuscrito.

\section{2 - Kelly Jackelini Jorge dos Santos}

Participação ativa na concepção e planejamento do projeto de pesquisa, obtenção, análise e interpretação dos dados, redação e revisão crítica do manuscrito.

\section{3 - Mariângela Monteiro Melo Baltazar}

Participação ativa na concepção e planejamento do projeto de pesquisa, obtenção, análise e interpretação dos dados, redação e revisão crítica do manuscrito.

\section{4 - Carlos Alexandre Molena Fernandes}

Participação ativa na concepção e planejamento do projeto de pesquisa e revisão crítica do manuscrito.

\section{5 - Aline Fernanda Junges Marques}

Participação ativa na revisão crítica do manuscrito.

\section{6 - Mateus Souza da Luz}

Participação ativa na revisão crítica do manuscrito.

\section{Como citar este artigo}

Matos FGOA, Santos KJJ, Baltazar MMM, Fernandes CAM, Marques AFJ, Luz MS. Perfil epidemiológico das fissuras labiopalatais de crianças atendidas em um centro de referência paranaense. Rev. Enferm. UFSM. 2020 [Acesso em: Anos Mês Dia]; vol. 10 e28: 1-14. DOI:https://doi.org/10.5902/2179769238654 